\title{
Model and algorithm for bilevel linear programming with fuzzy decision variables and multiple followers
}

\author{
Shengyue Deng ${ }^{\mathrm{a}, \mathrm{b}}$, Jintao Tan ${ }^{\mathrm{a}, *}$, Chengjie $\mathrm{Xu}^{\mathrm{a}}$, Xinfan Wang ${ }^{\mathrm{a}}$ \\ a School of Science, Hunan University of Technology, Zhuzhou, Hunan 412008, P. R. China. \\ ${ }^{b}$ Department of Mathematics and Computational Science, Xiangtan University, Xiangtan, Hunan 411105, P. R. China. \\ Communicated by $\mathrm{X}$. Liu
}

\begin{abstract}
The bilevel linear programming with fuzzy decision variables and multiple followers model (MFFVBLP) is firstly established and investigated, and the model optimal solution is shown to be equivalent to the optimal solution of the bilevel linear programming with multiple followers by using fuzzy structured element theory in this paper. The optimal solution of this model is found out by adopting the Kuhn-Tucker approach. An illustrative example is provided to demonstrate the feasibility and efficiency of the proposed method for solving the MFFVBLP model. (c)2017 All rights reserved.
\end{abstract}

Keywords: Bilevel linear programming, fuzzy decision variables, multiple followers, fuzzy structured element. 2010 MSC: 90C70, 93B40.

\section{Introduction}

Bilevel programming introduced by Von Stackelberg in 1952 [20] has been developed to solve the decentralized planning problems in which decision makers are often arranged within a hierarchical administrative structure. A bilevel programming problem occurs when two decision makers are located at different hierarchical levels. In general, a decision maker at the upper level is termed as the leader, and the lower level is termed as the follower [1,2]. In the context of bilevel programming, the leader first specifies a strategy, and then the follower specifies a strategy so as to optimize the objective with full knowledge of the action of the leader.

So far many researches on bilevel programming has centered on the linear version of the problem $[1,2,5,10,12,14]$. Two fundamental issues in theory and practice of bilevel programming problems are mostly concerned, one is how to model a real world bilevel programming, and the other is how to find properties and an optimal solution for the bilevel programming problem. There are many such hierarchical optimization problems in the fields of industry, agriculture, financial, transportation and so on $[4,6,9,21]$, but in many practical hierarchical decision making systems, resources, costs, demands, and

\footnotetext{
*Corresponding author

Email addresses: dsy110@163.com (Shengyue Deng), jeanette0219@163.com (Jintao Tan), xu-chengjie@163.com (Chengjie Xu), zzwxfydm@126. com (Xinfan Wang)

doi:10.22436/jnsa.010.04.68
} 
many other elements are often subject to fluctuations and are difficult to measure. Hence, it is necessary for us to formulate the decentralized decision-making problem with uncertainty as fuzzy models.

A fuzzy bilevel programming problem is a bilevel programming problem in which the coefficient, either in objective functions or in constraints, is described by fuzzy values. Sakawa et al. [12-17] formulated cooperative fuzzy bilevel programming problems and proposed an interactive fuzzy programming approach to solve the problems. From this approach, the concept of a bilevel programming was introduced based on fuzzy number $\lambda$-level sets. At the same time, some researches applied fuzzy set technique to deal with bilevel programming problems. Shih and Lee [18] applied fuzzy set theory to overcome the computational difficulties in solving bilevel problems. Sinha [19] started from the fuzzy mathematical programming approach to obtain the solution of multi-level linear programming problems. Recently, Zhang et al. [11, 23-25] studied fuzzy bilevel programming problem, with their focus on the situation that the leader or the follower had multiple objectives with fuzzy parameters and all followers shared their decision variables. They also provided some related algorithms based on the membership function in fuzzy set theory. Moreover, they have first solved the fuzzy linear bilevel programming problems with a specialized form of membership functions, triangular form, in the fuzzy parameters [5, 22]. However, fuzzy bilevel programming with fuzzy variables is still a new and challenging work for us.

This paper discusses a class of typical model of bilevel linear programming with fuzzy decision variables and multiple followers. Based on the homeomorphism properties between the bounded real fuzzy number and the monotone functions on $[-1,1]$, the comparison of a fuzzy number is changed into a new comparison of monotone function by the definition of fuzzy numbers structured element weighted order. Then the optimal solutions of new derived model is proved equivalent to the optimal solution of the bilevel linear programming with fuzzy decision variables and multiple followers. The feasibility of the proposed approach is further proved by giving a numerical example.

The following part of this paper is arranged as follows. Section 2 introduces some concepts and properties of the fuzzy numbers structured element weighted order. Section 3 studies the model and optimal solution of bilevel linear programming with fuzzy decision variables and multiple followers. Section 4 proposes an algorithm and demonstrates the efficiency of the algorithm by giving one numerical example. Finally, some conclusions are reached in Section 5.

\section{Preliminaries}

In this section, some necessary backgrounds and notions of fuzzy structured element theory are presented.

Definition 2.1 ([7]). Let $E$ be a fuzzy set on $R$ and $E(x)$ be the membership function of $E$. Then, $E$ is called a fuzzy structured element if

(1) $\mathrm{E}(0)=1$;

(2) $E(x)$ is monotonously increasing and right continuous on $[-1,0]$, monotone decreasing and left continuous on $(0,1]$;

(3) $\mathrm{E}(\mathrm{x})=0,(-\infty<x<-1$ or $1<x<+\infty)$.

Definition 2.2 ([7]). E is referred as a canonical fuzzy structured element if

(i) $\forall x \in(-1,1), \mathrm{E}(x)>0$;

(ii) $E(x)$ is continuous and strictly monotone increasing (decreasing) on $[-1,0]((0,1])$.

Definition $2.3([7])$. $E$ is called a symmetrical fuzzy structured element if $E(-x)=E(x)$.

Lemma 2.4 ([3]). Let $\mathrm{E}$ be a fuzzy structured element and $\mathrm{E}(\mathrm{x})$ be its membership function. Let the function $\mathrm{f}(\mathrm{x})$ be continuous and monotone on $[-1,1]$, then $f(E)$ is a fuzzy number, and the membership function of $f(E)$ is $E\left(f^{-1}(x)\right)$ (where $\mathrm{f}^{-1}(\mathrm{x})$ is rotational symmetry function for variable $\mathrm{x}$ and $\mathrm{y}$, if $\mathrm{f}$ is a strictly monotone function, then $\mathrm{f}^{-1}(\mathrm{x})$ is the inverse function of $f(x)$ ). 
Lemma 2.5 ([7]). For a given canonical fuzzy structured element $\mathrm{E}$ and any finite fuzzy number $\widetilde{\mathrm{A}}$, there always exists a monotone bounded function $f$ on $[-1,1]$, having the form $\widetilde{A}=f(E)$.

Definition $2.6([7])$. Let $\widetilde{A}=(a, b, c) \in \widetilde{N}_{c}(R)$ be a triangular fuzzy number, where $a \leqslant b \leqslant c$. The member function corresponding to $\widetilde{A}$ is defined by

$$
\mu_{\tilde{A}}(x)= \begin{cases}\frac{x-a}{b-a}, & a \leqslant x<b \\ 1, & x=b \\ \frac{x-c}{b-c}, & b<x \leqslant c \\ 0, & \text { others. }\end{cases}
$$

Lemma 2.7 ([7]). Let $\widetilde{\mathrm{A}}=(\mathrm{a}, \mathrm{b}, \mathrm{c})$ be the triangular fuzzy number and

$$
E(x)= \begin{cases}1+x, & -1 \leqslant x \leqslant 0 \\ 1-x, & 0<x \leqslant 1 \\ 0, & \text { others }\end{cases}
$$

be the triangular fuzzy structured element. Then we have

$$
f(x)= \begin{cases}(b-a) x+b, & -1 \leqslant x \leqslant 0 \\ (c-b) x+b, & 0<x \leqslant 1 \\ 0, & \text { others }\end{cases}
$$

from which we can easily get $\widetilde{A}=\mathrm{f}(\mathrm{E})$.

Next we denote all bounded fuzzy numbers on $R$ by $\widetilde{N}_{c}(R)$. Let mapping $H_{E}: B_{[-1,1]} \rightarrow \widetilde{N}_{c}(R)$ be given by

$$
f \rightarrow H_{E}(f)=f(E) \in \widetilde{N}_{c}(R) .
$$

We say $H_{E}$ is a fuzzy function induced by structured element $E$ on $B_{[-1,1]}$. It is easily known that $H_{E}$ is a one-to-one isometric mapping from $B_{[-1,1]}$ onto $\widetilde{N}_{c}(R)$ ([8]).

Definition 2.8 ([3]). Let $\widetilde{A}_{1}, \widetilde{A}_{2} \in \widetilde{N}_{C}(R)$. Their structured element representations are $\widetilde{A}_{i}=f_{i}(E), i=1,2$, respectively, where $E$ is given a canonical fuzzy structured element whose membership function is $E(x)$. Let $f_{1}(x)$ and $f_{2}(x)$ be the same sequence monotonic functions on $[-1,1]$ (function with the same monotone property). Then from

$$
\widetilde{A}_{1} \leqslant \widetilde{A}_{2} \Leftrightarrow F\left(\widetilde{A}_{1}, \widetilde{A}_{2}\right)=\int_{-1}^{1} E(x)\left(f_{1}(x)-f_{2}(x)\right) d x \leqslant 0
$$

we may define a total order relation " $\leqslant$ " on $\widetilde{N}_{C}(R)$ which is called the structured element weighted order for fuzzy numbers.

Lemma 2.9 ([7]). Let $\mathrm{E}$ be a symmetrical fuzzy structured element. Let $\mathrm{f}_{1}(\mathrm{x})$ and $\mathrm{f}_{2}(\mathrm{x})$ be the same sequence monotonic functions on $[-1,1]$. Let fuzzy numbers $\widetilde{A}_{1}=f_{1}(E)$, and $\widetilde{A}_{2}=f_{2}(E)$. Then

$$
\widetilde{A}_{1}+\widetilde{A}_{2}=f_{1}(E)+f_{2}(E), \quad \widetilde{A}_{1}-\widetilde{A}_{2}=f_{1}(E)+f_{2}^{\tau}(E), \quad k \widetilde{A}_{1}=|k| f_{1}^{\tau}(E) .
$$

It readily follows from the last equality that $\mathrm{f}_{1}^{\tau}(E)=\mathrm{f}_{1}(\mathrm{E})$ for $\mathrm{k} \geqslant 0$ and $\mathrm{f}_{1}^{\tau}(\mathrm{E})=-\mathrm{f}_{1}(-\mathrm{E})$ for $\mathrm{k}<0$.

Lemma 2.10 ([7]). Let $\mathrm{f}$ be a monotonously bounded function on $[-1,1]$. Given $\mathrm{E}$ is a fuzzy structured element on $\mathrm{R}$ which yields the fuzzy number $\widetilde{\mathrm{A}}=\mathrm{f}(\mathrm{E})$. For any $\lambda \in[0,1]$, the $\lambda$-level set of $\mathrm{E}$ is denoted as $\mathrm{E}_{\lambda}=\left[\mathrm{e}_{\lambda}^{-}, \mathrm{e}_{\lambda}^{+}\right]$, where $e_{\lambda}^{-} \in[-1,0]$ and $e_{\lambda}^{+} \in[0,1]$. If $f$ is a monotone increasing function on $[-1,1]$, then

$$
A_{\lambda}=[f(E)]_{\lambda}=f\left(E_{\lambda}\right)=\left[f\left(e_{\lambda}^{-}\right), f\left(e_{\lambda}^{+}\right)\right] .
$$

If $\mathrm{f}$ is a monotone decreasing function on $[-1,1]$, then

$$
A_{\lambda}=\left[f\left(e_{\lambda}^{+}\right), f\left(e_{\lambda}^{-}\right)\right] .
$$

The proof of above lemmas can be found in reference $[3,7,8]$. 


\section{Bilevel linear programming with fuzzy decision variables and multiple followers model}

Consider the following bilevel linear programming with fuzzy decision variables and multiple followers (MFFVBLP).

$$
\left\{\begin{array}{l}
\min _{\widetilde{x}_{i}} \widetilde{Z}_{1}^{1}=\sum_{i=1}^{N} c_{i}^{1} \widetilde{x}_{i}+\sum_{s=1}^{S} \sum_{j=1}^{M} d_{s j}^{1} \widetilde{y}_{s j} ; \\
\text { s.t. where } \widetilde{y}_{s j} \text { is the lower level problem's solution: } \\
\min _{\widetilde{y}_{s j}} \widetilde{Z}_{s}^{2}=\sum_{i=1}^{N} c_{s i}^{2} \widetilde{x}_{i}+\sum_{j=1}^{M} d_{s j}^{2} \widetilde{y}_{s j}, \\
\text { s.t. } \sum_{i=1}^{N} a_{t i}^{s} \widetilde{x}_{i}+\sum_{j=1}^{M} b_{t j}^{s} \widetilde{y}_{j} \leqslant \widetilde{e}_{t}^{s}, \\
\widetilde{x}_{i} \geqslant 0 ; \widetilde{y}_{s j} \geqslant 0, \\
i=1,2, \cdots, N ; j=1,2, \cdots, M ; s=1,2, \cdots, S ; t=1,2, \cdots, T,
\end{array}\right.
$$

where $\widetilde{x}_{i} \in R, \widetilde{y}_{s j} \in R, \widetilde{e}_{t}^{s} \in \widetilde{N}_{C}(R) ; c_{i}^{1}, c_{s i}^{2}, d_{s j}^{1}, d_{s j}^{2}, a_{t i}^{s}, b_{t j}^{s} \in R$.

The following theorem indicates that MFFVBLP (3.1) may be transformed another equivalent representation that lays the basis for the algorithm in next section.

Theorem 3.1. Let $\tilde{x}_{i}=\left(\underline{x}_{i}, x_{i}, \bar{x}_{i}\right)$ and $\tilde{y}_{s j}=\left(\underline{y}_{s j}, y_{s j}, \bar{y}_{s j}\right)$ be triangular fuzzy numbers for $i=1,2, \cdots, N$; $j=1,2, \cdots, M$ and $s=1,2, \cdots, S$. Let $\widetilde{Z}_{1}^{1}=G_{1}^{1}(E), \widetilde{Z}_{s}^{2}=G_{s}^{2}(E), \widetilde{x}_{i}=f_{i}(E), \widetilde{y}_{s j}=F_{s j}(E), \widetilde{e}_{t}^{s}=\psi_{t}^{s}(E)$, $\mathrm{M}_{1}^{1}=\int_{-1}^{1} \mathrm{E}(\mathrm{t}) \mathrm{G}_{1}^{1}(\mathrm{t}) \mathrm{dt}$. Suppose that $\mathrm{E}$ is a canonical symmetrical fuzzy structured element and $\mathrm{G}_{1}^{1}(\mathrm{t}), \mathrm{G}_{\mathrm{s}}^{2}(\mathrm{t})$, $f_{i}(t), F_{s j}(t)$ and $\psi_{t}^{s}(t)$ are monotonous increasing functions for $i=1,2, \cdots, N, j=1,2, \cdots, M, s=1,2, \cdots, S$ and $\mathrm{t}=1,2, \cdots, \mathrm{T}$. Then the model (3.1) is equivalent to the following model

$$
\left\{\begin{array}{l}
\min _{\underline{x}_{i}, x_{i}, \bar{x}_{i}} M_{1}^{1}=\sum_{i=1}^{N} c_{i}^{1} \int_{-1}^{1} E(t) f_{i}(t) d t+\sum_{s=1}^{S} \sum_{j=1}^{M} d_{s j}^{1} \int_{-1}^{1} E(t) F_{s j}(t) d t ; \\
\text { s.t. where } \underline{y}_{s j^{\prime}}, y_{s j}, \bar{y}_{s j} \text { is the lower level problem's solution: } \\
\min _{\underline{y}_{s,} y_{s j}, \bar{y}_{s j}} M_{s}^{2}=\sum_{i=1}^{N} c_{s i}^{2} \int_{-1}^{1} E(t) f_{i}(t) d t+\sum_{j=1}^{M} d_{s j}^{2} \int_{-1}^{1} E(t) F_{s j}(t) d t, \\
\text { s.t. } \sum_{i=1}^{N} a_{t i}^{s} \int_{-1}^{1} E(t) f_{i}(t) d t+\sum_{j=1}^{M} b_{t j}^{s} \int_{-1}^{1} E(t) F_{s j}(t) d t \leqslant \int_{-1}^{1} E(t) \psi_{t}^{s}(t) d t, \\
\bar{x}_{i}-x_{i} \geqslant 0, x_{i}-\underline{x}_{i} \geqslant 0, \bar{y}_{s j}-y_{s j} \geqslant 0, y_{s j}-\underline{y}_{s j} \geqslant 0, f_{i}(-1) \geqslant 0, F_{s j}(-1) \geqslant 0, \\
i=1,2, \cdots, N ; j=1,2, \cdots, M ; s=1,2, \cdots, S ; t=1,2, \cdots, T .
\end{array}\right.
$$

Proof. By Definition 2.8 and Lemma 2.7, it follows that scaling the fuzzy number $\widetilde{Z}_{1}^{1}$ is equivalent to scale $M_{1}^{1}=\int_{-1}^{1} E(t) G_{1}^{1}(t) d t$ in the model (3.1). So we have

$$
\widetilde{Z}_{1}^{1}=G_{1}^{1}(E)=\sum_{i=1}^{N} c_{i}^{1} \widetilde{x}_{i}+\sum_{s=1}^{S} \sum_{j=1}^{M} d_{s j}^{1} \widetilde{y}_{s j}=\sum_{i=1}^{N}\left|c_{i}^{1}\right| f_{i}^{\tau}(E)+\sum_{s=1}^{S} \sum_{j=1}^{M}\left|d_{s j}^{1}\right| F_{s j}^{\tau}(E) .
$$

By the use of fuzzy structured element theory, we have

$$
\begin{aligned}
M_{1}^{1}=\int_{-1}^{1} E(t) G_{1}^{1}(t) d t & =\int_{-1}^{1} E(t)\left[\sum_{i=1}^{N}\left|c_{i}^{1}\right| f_{i}^{\tau}(t)+\sum_{s=1}^{S} \sum_{j=1}^{M}\left|d_{s j}^{1}\right| F_{s j}^{\tau}(t)\right] d t \\
& =\int_{-1}^{1} E(t) \sum_{i=1}^{N}\left|c_{i}^{1}\right| f_{i}^{\tau}(t) d t+\int_{-1}^{1} E(t) \sum_{s=1}^{s} \sum_{j=1}^{M}\left|d_{s j}^{1}\right| F_{s j}^{\tau}(t) d t \\
& =\sum_{i=1}^{N}\left|c_{i}^{1}\right| \int_{-1}^{1} E(t) f_{i}^{\tau}(t) d t+\sum_{s=1}^{S} \sum_{j=1}^{M}\left|d_{s j}^{1}\right| \int_{-1}^{1} E(t) F_{s j}^{\tau}(t) d t .
\end{aligned}
$$


First, we take the item $\sum_{i=1}^{N}\left|c_{i}^{1}\right| \int_{-1}^{1} E(t) f_{i}^{\tau}(t) d t$ into account. Actually, it follows from Lemma 2.9 that

$$
\sum_{i=1}^{N}\left|c_{i}^{1}\right| \int_{-1}^{1} E(t) f_{i}^{\tau}(t) d t=\sum_{i=1}^{N} c_{i}^{1} \int_{-1}^{1} E(t) f_{i}(t) d t,
$$

when $c_{i}^{1} \geqslant 0$ and

$$
\sum_{i=1}^{N}\left|c_{i}^{1}\right| \int_{-1}^{1} E(t) f_{i}^{\tau}(t) d t=-\sum_{i=1}^{N} c_{i}^{1} \int_{-1}^{1} E(t)\left(-f_{i}(t)\right) d t,
$$

when $c_{i}^{1}<0$. Since $E$ is symmetrical structured element, we have $E(-t)=E(t)$. By using the transform element method of the definite integral, it holds that

$$
\sum_{i=1}^{N}\left|c_{i}^{1}\right| \int_{-1}^{1} E(t) f_{i}^{\tau}(t) d t=\sum_{i=1}^{N} c_{i}^{1} \int_{-1}^{1} E(t) f_{i}(t) d t .
$$

Analogously, another item in $M_{1}^{1}$ could be rewritten as

$$
\sum_{s=1}^{S} \sum_{j=1}^{M}\left|d_{s j}^{1}\right| \int_{-1}^{1} E(t) F_{s j}^{\tau}(t) d t=\sum_{s=1}^{S} \sum_{j=1}^{M} d_{s j}^{1} \int_{-1}^{1} E(t) F_{s j}(t) d t
$$

which results in

$$
M_{1}^{1}=\sum_{i=1}^{N} c_{i}^{1} \int_{-1}^{1} E(t) f_{i}(t) d t+\sum_{s=1}^{S} \sum_{j=1}^{M} d_{s j}^{1} \int_{-1}^{1} E(t) F_{s j}(t) d t .
$$

On the other hand, we similarly have

$$
M_{s}^{2}=\sum_{i=1}^{N} c_{s i}^{2} \int_{-1}^{1} E(t) f_{i}(t) d t+\sum_{j=1}^{M} d_{s j}^{2} \int_{-1}^{1} E(t) F_{s j}(t) d t .
$$

By Lemma 2.7 and Lemma 2.9, we obtain the constraint

$$
\sum_{i=1}^{N} a_{t i}^{s} \int_{-1}^{1} E(t) f_{i}(t) d t+\sum_{j=1}^{M} b_{t j}^{s} \int_{-1}^{1} E(t) F_{s j}(t) d t \leqslant \int_{-1}^{1} E(t) \psi_{t}^{s}(t) d t
$$

Since for $i=1,2, \cdots, N, j=1,2, \cdots, M$, and $s=1,2, \cdots, S, \widetilde{x}_{i}=\left(\underline{x}_{i}, x_{i}, \bar{x}_{i}\right)$ and $\widetilde{y}_{s j}=\left(\underline{y}_{s j}, y_{s j}, \bar{y}_{s j}\right)$ are triangular fuzzy numbers, we know from Definition 2.8 that $\bar{x}_{i}-x_{i} \geqslant 0, x_{i}-\underline{x}_{i} \geqslant 0, \bar{y}_{s j}-y_{s j} \geqslant 0$, and $y_{s j}-\underline{y}_{s j} \geqslant 0$. Also, it follows from Definition 2.1 and Lemma 2.10 that $E_{\lambda}=\left[e_{\lambda}^{-}, e_{\lambda}^{+}\right]$with $e_{\lambda}^{-} \in[-1,0]$ and $e_{\lambda}^{+} \in[0,1]$. By assuming that $f_{i}(t)$ and $F_{s j}(t)$ are both monotonous increasing functions on $[-1,1]$, we then have

$$
\left(\widetilde{x}_{i}\right)_{\lambda}=\left[f_{i}(E)\right]_{\lambda}=f_{i}\left(E_{\lambda}\right)=f_{i}\left(e_{\lambda}^{-}, e_{\lambda}^{+}\right)=\left[f_{i}\left(e_{\lambda}^{-}\right), f_{i}\left(e_{\lambda}^{+}\right)\right]
$$

and

$$
\left(\widetilde{y}_{s j}\right)_{\lambda}=\left[F_{s j}(E)\right]_{\lambda}=F_{s j}\left(E_{\lambda}\right)=F_{s j}\left(e_{\lambda}^{-}, e_{\lambda}^{+}\right)=\left[F_{s j}\left(e_{\lambda}^{-}\right), F_{s j}\left(e_{\lambda}^{+}\right)\right] .
$$

This together with $\widetilde{x}_{i} \geqslant 0$ and $\widetilde{y}_{s j} \geqslant 0$ indicate

$$
f_{i}(-1) \leqslant f_{i}\left(e_{\lambda}^{-}\right) \leqslant f_{i}\left(e_{\lambda}^{+}\right) \leqslant f_{i}(1)
$$

and

$$
F_{s j}(-1) \leqslant F_{s j}\left(e_{\lambda}^{-}\right) \leqslant F_{s j}\left(e_{\lambda}^{+}\right) \leqslant F_{s j}(1) .
$$

So we have $\left[f_{i}(-1), f_{i}(1)\right] \geqslant 0$ and $\left[F_{s j}(-1), F_{s j}(1)\right] \geqslant 0$ which further yields $f_{i}(-1) \geqslant 0$ and $F_{s j}(-1) \geqslant 0$. The proof is completed. 


\section{Algorithm and numerical example}

We list all steps of the algorithm for solving the proposed MFFVBLP model. Note that it is derived from the result of Theorem 3.1.

\subsection{Algorithm}

1. Given that the fuzzy number is triangular, we have $\mu_{\widetilde{A}}=E\left(f^{-1}(t)\right)$, according to Lemma 2.7 and the expression of E. Compute $f_{i}(t), F_{s j}(t)$ and $\psi_{t}^{s}(t)$.

2. Calculate integrals $\int_{-1}^{1} E(t) f_{i}(t) d t, \int_{-1}^{1} E(t) F_{s j}(t) d t$ and $\int_{-1}^{1} E(t) \psi_{t}^{s}(t) d t$. Then plug them into model (3.2).

3. According to Theorem 3.1, the MFFVBLP model is transformed into the classical bilevel linear programming with multiple followers model (3.2). Compute the optimal solution of the model (3.2) via the Kuhn-Tucker's approach ([10]).

4. Insert the derived optimal solution of model (3.2) into model (3.1). We then get the optimal solution of the original MFFVBLP model.

We provide an illustratively numerical example in this part to demonstrate the feasibility and efficiency of the proposed method for solving the MFFVBLP problem.

\subsection{Example}

Let $\widetilde{x}_{1}=\left(\underline{x}_{1}, x_{1}, \bar{x}_{1}\right), \widetilde{x}_{2}=\left(\underline{x}_{2}, x_{2}, \bar{x}_{2}\right), \widetilde{y}_{11}=\left(\underline{y}_{11}, y_{11}, \bar{y}_{11}\right)$ and $\widetilde{y}_{21}=\left(\underline{y}_{21}, y_{21}, \bar{y}_{21}\right)$ be the leader decision variable and the followers decision variables, respectively. Let $\widetilde{Z}_{1}^{1}$ and $\widetilde{Z}_{1}^{2}, \widetilde{Z}_{2}^{2}$ be objective functions of the leader and the followers. Construct the following MFFVBLP problem.

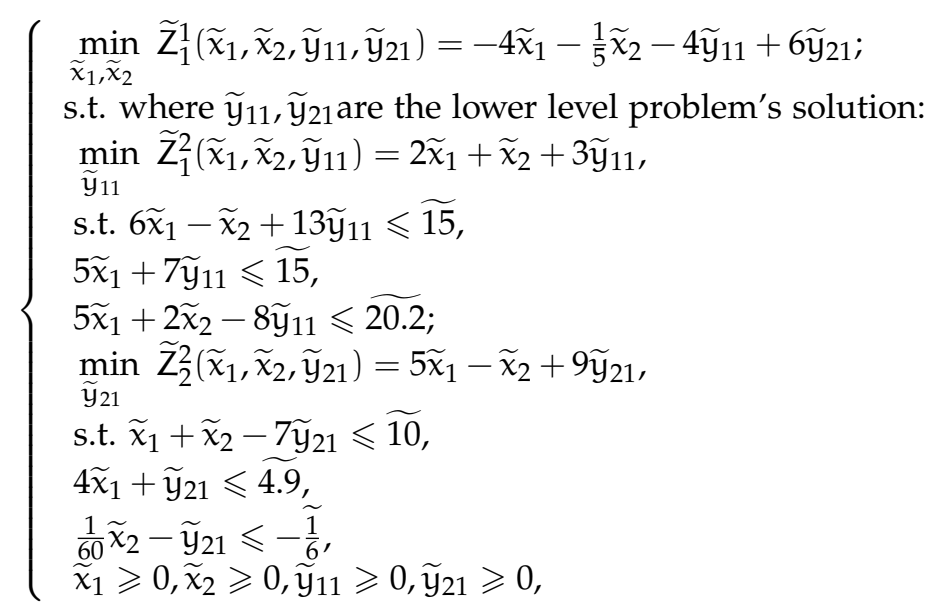

where the triangular fuzzy numbers are $\frac{\widetilde{1}}{6}=\left(\frac{2}{15}, \frac{1}{6}, \frac{1}{5}\right), \widetilde{4.9}=(4.5,4.9,5.9), \widetilde{10}=(8,10,12), \widetilde{15}=$ $(14.5,15,15.5), \widetilde{20.2}=(18.7,20.2,20.5)$.

1. By Lemma 2.7 and Theorem 3.1, we have

$$
\begin{aligned}
& \psi_{1}^{1}(t)=\psi_{2}^{1}(t)= \begin{cases}2 t+15, & -1 \leqslant t \leqslant 0 \\
2 t+15, & 0 \leqslant t \leqslant 1 \\
0, & \text { others }\end{cases} \\
& \psi_{3}^{1}(t)= \begin{cases}1.5 t+20.2, & -1 \leqslant t \leqslant 0 \\
0.3 t+20.2, & 0 \leqslant t \leqslant 1 \\
0, & \text { others }\end{cases} \\
& \psi_{1}^{2}(t)=\left\{\begin{array}{cl}
2 t+10, & -1 \leqslant t \leqslant 0, \\
2 t+10, & 0 \leqslant t \leqslant 1, \\
0, & \text { others, }
\end{array}\right. \\
& \psi_{2}^{21}(t)= \begin{cases}0.4 t+4.9, & -1 \leqslant t \leqslant 0 \\
t+4.9, & 0 \leqslant t \leqslant 1 \\
0, & \text { others, }\end{cases} \\
& \psi_{3}^{2}(t)= \begin{cases}\frac{1}{30} t+\frac{1}{6}, & -1 \leqslant t \leqslant 0 \\
\frac{1}{30} t+\frac{1}{6}, & 0 \leqslant t \leqslant 1 \\
0, & \text { others }\end{cases} \\
& f_{1}(t)= \begin{cases}\left(x_{1}-\underline{x}_{1}\right) t+x_{1}, & -1 \leqslant t \leqslant 0 \\
\left(\bar{x}_{1}-x_{1}\right) t+x_{1}, & 0 \leqslant t \leqslant 1 \\
0, & \text { others }\end{cases}
\end{aligned}
$$




$$
\begin{aligned}
f_{2}(t) & = \begin{cases}\left(x_{2}-\underline{x}_{2}\right) t+x_{2}, & -1 \leqslant t \leqslant 0, \\
\left(\bar{x}_{2}-x_{2}\right) t+x_{2}, & 0 \leqslant t \leqslant 1, \\
0, & \text { others, }\end{cases} \\
F_{21}(t) & = \begin{cases}\left(y_{21}-\underline{y}_{21}\right) t+y_{21}, & -1 \leqslant t \leqslant 0 \\
\left(\bar{y}_{21}-y_{21}\right) t+y_{21}, & 0 \leqslant t \leqslant 1 \\
0, & \text { others. }\end{cases}
\end{aligned}
$$

2. Compute integrals

$$
\begin{array}{rlrl}
\int_{-1}^{1} E(t) \psi_{1}^{1}(t) d t & =\int_{-1}^{1} E(t) \psi_{2}^{1}(t) d t=15, & & \int_{-1}^{1} E(t) \psi_{3}^{1}(t) d t=20 \\
\int_{-1}^{1} E(t) \psi_{1}^{2}(t) d t & =10, & \int_{-1}^{1} E(t) \psi_{2}^{2}(t) d t & =5 \\
\int_{-1}^{1} E(t) \psi_{3}^{2}(t) d t & =\frac{1}{6}, & \int_{-1}^{1} E(t) f_{1}(t) d t & =\frac{1}{6}\left(\underline{x}_{1}+4 x_{1}+\bar{x}_{1}\right), \\
\int_{-1}^{1} E(t) f_{2}(t) d t & =\frac{1}{6}\left(\underline{x}_{2}+4 x_{2}+\bar{x}_{2}\right), & \int_{-1}^{1} E(t) F_{11}(t) d t & =\frac{1}{6}\left(\underline{y}_{11}+4 y_{11}+\bar{y}_{11}\right), \\
\int_{-1}^{1} E(t) F_{21}(t) d t & =\frac{1}{6}\left(\underline{y}_{21}+4 y_{21}+\bar{y}_{21}\right), &
\end{array}
$$

and $x_{1}-\underline{x}_{1} \geqslant 0, \bar{x}_{1}-x_{1} \geqslant 0, x_{2}-\underline{x}_{2} \geqslant 0, \bar{x}_{2}-x_{2} \geqslant 0, y_{11}-\underline{y}_{11} \geqslant 0, \bar{y}_{11}-y_{11} \geqslant 0, y_{21}-\underline{y}_{21} \geqslant 0$, $\bar{y}_{21}-y_{21} \geqslant 0, f_{1}(-1)=\underline{x}_{1}, f_{2}(-1)=\underline{x}_{2}, F_{11}(-1)=\underline{y}_{11}, F_{21}(-1)=\underline{y}_{21}$.

3. By Theorem 3.1, the original problem is equivalent to the following problem of bilevel linear programming with multiple followers.

$$
\left\{\begin{array}{l}
\min _{\underline{x}_{1}, x_{1}, \bar{x}_{1}, \underline{x}_{2}, x_{2}, \bar{x}_{2} \in R} M_{1}^{1}=-\frac{2}{3}\left(\underline{x}_{1}+4 x_{1}+\bar{x}_{1}\right)-\frac{1}{30}\left(\underline{x}_{2}+4 x_{2}+\bar{x}_{2}\right)-\frac{2}{3}\left(\underline{y}_{11}+4 y\right. \\
\quad+\underline{y}_{21}+4 y_{21}+\bar{y}_{21} ; \\
\text { s.t. where } \underline{y}_{11}, y_{11}, \bar{y}_{11}, \underline{y}_{21}, y_{21}, \bar{y}_{21} \text { are the lower level problem's solution: } \\
\quad \min _{\underline{y}_{11}, y_{11}, \bar{y}_{11}} M_{1}^{2}=\frac{1}{3}\left(\underline{x}_{1}+4 x_{1}+\bar{x}_{1}\right)+\frac{1}{6}\left(\underline{x}_{2}+4 x_{2}+\bar{x}_{2}\right)+\frac{1}{2}\left(\underline{y}_{11}+4 y_{11}+\bar{y}_{11}\right) ; \\
\text { s.t. } \underline{x}_{1}+4 x_{1}+\bar{x}_{1}-\frac{1}{6}\left(\underline{x}_{2}+4 x_{2}+\bar{x}_{2}\right)+\frac{13}{6}\left(\underline{y}_{11}+4 y_{11}+\bar{y}_{11}\right) \leqslant 15 \\
\frac{5}{6}\left(\underline{x}_{1}+4 x_{1}+\bar{x}_{1}\right)+\frac{7}{6}\left(\underline{y}_{11}+4 y_{11}+\bar{y}_{11}\right) \leqslant 15 \\
\frac{5}{6}\left(\underline{x}_{1}+4 x_{1}+\bar{x}_{1}\right)+\frac{1}{3}\left(\underline{x}_{2}+4 x_{2}+\bar{x}_{2}\right)-\frac{4}{3}\left(\underline{y}_{11}+4 y_{11}+\bar{y}_{11}\right) \leqslant 20 \\
\quad \min _{21} M_{2}^{2}=\frac{5}{6}\left(\underline{x}_{1}+4 x_{1}+\bar{x}_{1}\right)-\frac{1}{6}\left(\underline{x}_{2}+4 x_{2}+\bar{x}_{2}\right)+\frac{2}{3}\left(\underline{y}_{21}+4 y_{21}+\bar{y}_{21}\right) \\
\text { s.t. } \frac{1}{6}\left(\underline{x}_{1}+4 x_{1}+\bar{x}_{1}\right)+\frac{1}{6}\left(\underline{x}_{2}+4 x_{2}+\bar{x}_{2}\right)-\frac{7}{6}\left(\underline{y}_{21}+4 y_{21}+\bar{y}_{21}\right) \leqslant 10 \\
\frac{2}{3}\left(\underline{x}_{1}+4 x_{1}+\bar{x}_{1}\right)+\frac{1}{6}\left(\underline{y}_{21}+4 y_{21}+\bar{y}_{21}\right) \leqslant 5 \\
\frac{1}{360}\left(\underline{x}_{2}+4 x_{2}+\bar{x}_{2}\right)-\frac{1}{6}\left(\underline{y}_{21}+4 y_{21}+\bar{y}_{21}\right) \leqslant-\frac{1}{6}, \\
x_{1}-\underline{x}_{1} \geqslant 0, \bar{x}_{1}-x_{1} \geqslant 0, x_{2}-\underline{x}_{2} \geqslant 0, \bar{x}_{2}-x_{2} \geqslant 0, \\
y_{11}-\underline{y}_{11} \geqslant 0, \bar{y}_{11}-\underline{y}_{11} \geqslant 0, y_{21}-\underline{y}_{21} \geqslant 0, \bar{y}_{21}-y_{21} \geqslant 0 \\
\underline{x}_{1} \geqslant 0, \underline{x}_{2} \geqslant 0, \underline{y}_{11} \geqslant 0, \underline{y}_{21} \geqslant 0 .
\end{array}\right.
$$


4. Now we exploit the Kuhn-Tucker approach to get an optimal solution of model (4.2) and plug them into model (4.1). We have

$$
\begin{aligned}
\left(\underline{x}_{1}, x_{1}, \bar{x}_{1}\right) & =(0.1930,1.2585,1.4799), \\
\left(\underline{x}_{2}, x_{2}, \bar{x}_{2}\right) & =(5.0616,10.7876,27.2863), \\
\left(\underline{y}_{11}, y_{11}, \bar{y}_{11}\right) & =(0.4197,1.0177,3.5761), \\
\left(\underline{y}_{21}, \underline{y}_{21}, \bar{y}_{21}\right) & =(0.1724,0.4090,1.3637), \\
M_{1}^{1} & =-9.1934, M_{1}^{2}=18.8519, M_{2}^{2}=-2.2357, \\
\widetilde{z}_{1}^{1} & =\left(\underline{z}_{1}^{1}, z_{1}^{1}, \bar{z}_{1}^{1}\right)=(-25.9564,-8.5958,5.1797), \\
\widetilde{z}_{1}^{2} & =\left(\underline{z}_{1}^{2}, z_{1}^{2}, \bar{z}_{1}^{2}\right)=(6.7481,16.2468,41.3763), \\
\widetilde{z}_{2}^{2} & =\left(\underline{z}_{2}^{2}, z_{2}^{2}, \bar{z}_{2}^{2}\right)=(-24.1624,-1.3494,16.1460) .
\end{aligned}
$$

\section{Conclusions}

A real-world bilevel decision problem may be modeled to have fuzzy coefficients. This paper has investigated the bilevel linear programming with fuzzy decision variables and multiple followers model and solved this complex problem by using the fuzzy structured element method. Further study includes the development of models and methods for fuzzy multilevel programming. We will also explore effective applications of the proposed techniques.

\section{Acknowledgment}

The work was supported in part by the General Project of Hunan Provincial Natural Science Foundation of China (Grant nos.2016JJ2043, 2017JJ2071 and 2015JJ2047), the National Natural Science Foundation of China (Grant nos. 11571292 and 11471279), the General Project of Hunan Provincial Education Department of China (16C0472), and the Scientific Research Key Project of the Higher Education Institutions of Hunan Province of China (Grant nos. 15A055 and 2017B075).

\section{References}

[1] W. F. Bialas, M. H. Karwan, Two-level linear programming, Management Sci., 30 (1984), 1004-1020. 1

[2] S. Dempe, Foundations of bilevel programming, Nonconvex Optimization and its Applications, Kluwer Academic Publishers, Dordrecht, (2002). 1

[3] S.-Y. Deng, L.-Q. Zhou, X.-F. Wang, Bi-level multiple followers linear programming with upper constraint and fuzzy decision variables, Control Decis., 29 (2014), 1803-1808. 2.4, 2.8, 2

[4] S.-Y. Deng, L.-Q. Zhou, X.-F. Wang, Solving the fuzzy bilevel linear programming with multiple followers through structured element method, Math. Probl. Eng., 2014 (2014), 6 pages. 1

[5] Y. Gao, G. Zhang, J. Ma, J. Lu, A ג-cut and goal-programming-based algorithm for fuzzy-linear multiple-objective bilevel optimization, IEEE Trans. Fuzzy Syst., 18 (2010), 1-13. 1

[6] H. A. Gil, F. D. Galiana, E. L. Da Silva, Nodal price control: a mechanism for transmission network cost allocation, IEEE Trans. Power Syst., 21 (2006), 3-10. 1

[7] S.-Z. Guo, Principle of fuzzy mathematical analysis based on structured element, Northeast University Press, Shenyang, China, (2004). 2.1, 2.2, 2.3, 2.5, 2.6, 2.7, 2.9, 2.10, 2

[8] S.-Z. Guo, Comparison and ordering of fuzzy numbers based on method of structured element, Syst. Eng. Theory Pract., 29 (2009), 106-111. 2, 2

[9] K. Kogan, C. S. Tapiero, Optimal co-investment in supply chain infrastructure, European J. Oper. Res., 192 (2009), 265-276. 1

[10] J. Lu, C.-G. Shi, G.-Q. Zhang, On bilevel multi-follower decision making: general framework and solutions, Inform. Sci., 176 (2006), 1607-1627. 1, 3

[11] J. Lu, G.-Q. Zhang, T. Dillon, Fuzzy multi-objective bilevel decision making by an approximation Kth-best approach, J. Mult.-Valued Logic Soft Comput., 14 (2008), 205-232. 1

[12] M. Sakawa, H. Katagiri, T. Matsui, Stackelberg solutions for fuzzy random two-level linear programming through probability maximization with possibility, Fuzzy Sets and Systems, 188 (2012), 45-57. 1 
[13] M. Sakawa, I. Nishizaki, Interactive fuzzy programming for decentralized two-level linear programming problems, Theme: decision and optimization, Fuzzy Sets and Systems, 125 (2001), 301-315.

[14] M. Sakawa, I. Nishizaki, Interactive fuzzy programming for two-level linear fractional programming problems, Optimization and decision, Fuzzy Sets and Systems, 119 (2001), 31-40. 1

[15] M. Sakawa, I. Nishizaki, Interactive fuzzy programming for two-level nonconvex programming problems with fuzzy parameters through genetic algorithms, Theme: optimization and decision support systems, Fuzzy Sets and Systems, 127 (2002), 185-197.

[16] M. Sakawa, I. Nishizaki, Y. Uemura, Interactive fuzzy programming for multi-level linear programming problems with fuzzy parameters, Fuzzy Sets and Systems, 109 (2000), 3-19.

[17] M. Sakawa, K. Yauchi, Interactive decision making for multiobjective nonconvex programming problems with fuzzy numbers through coevolutionary genetic algorithms, Fuzzy Sets and Systems, 114 (2000), 151-165. 1

[18] H.-S. Shih, E. S. Lee, Compensatory fuzzy multiple level decision making, Fuzzy Sets and Systems, 114 (2000), 71-87. 1

[19] S. Sinha, Fuzzy programming approach to multi-level programming problems, Fuzzy Sets and Systems, 136 (2003), 189-202. 1

[20] H. van Stackelberg, The theory of the market economy, Oxford University Press, Oxford, (1952). 1

[21] Z. Yao, S. C. H. Leung, K. K. Lai, Manufacturer's revenue-sharing contract and retail competition, European J. Oper. Res., 186 (2008), 637-651. 1

[22] G.-Q. Zhang, J. Lu, Model and approach of fuzzy bilevel decision making for logistics planning problem, J. Enterp. Inf. Manag., 20 (2007), 178-197. 1

[23] G.-Q. Zhang, J. Lu, T. Dillon, Decentralized multi-objective bilevel decision making with fuzzy demands, Knowl. Based Syst., 20 (2007), 495-507. 1

[24] G.-Q. Zhang, J. Lu, Y. Gao, An algorithm for fuzzy multi-objective multi-follower partial cooperative bilevel programming, J. Intell. Fuzzy Syst., 19 (2008), 303-319.

[25] G.-Q. Zhang, J. Lu, Y. Gao, Fuzzy bilevel programming: multi-objective and multi-follower with shared variables, Int. J. Uncertain., Fuzz., 16 (2008), 105-133. 1 